\title{
Predictive factors of contrast-enhanced ultrasonography for the response to transarterial chemoembolization in hepatocellular carcinoma
}

Kil Hyo Park', Soon Ha Kwon', Yong Sub Lee', Soung Won Jeong', Jae Young Jang', Sae Hwan Lee', Sang Gyune Kim', Sang-Woo Cha', Young Seok Kim', Young Deok Cho', Hong Soo Kim', Boo Sung Kim', and Yong Jae Kim²

Institute for Digestive Research, Digestive Disease Center, 'Department of Internal Medicine, ${ }^{2}$ Department of Radiology, Soonchunhyang University Hospital, Seoul, Korea

Background/Aims: The predictive role of contrast-enhanced ultrasonography (CEUS) before performing transarterial chemoembolization (TACE) has not been determined. We assessed the possible predictive factors of CEUS for the response to TACE.

Methods: Seventeen patients with 18 hepatocellular carcinoma (HCC) underwent TACE. All of the tumors were studied with CEUS before TACE using a second-generation ultrasound contrast agent (SonoVue ${ }^{\oplus}$, Bracco, Milan, Italy). The tumor response to TACE was classified with a score between 1 and 4 according to the remaining enhancing-tumor percentage based on modified response evaluation criteria in solid tumors (mRECIST): 1, enhancing tumor $<25 \% ; 2,25 \% \leq$ enhancing tumor $<50 \% ; 3,50 \% \leq$ enhancing tumor $<75 \%$; and 4 , enhancing tumor $\geq 75 \%$ ). A score of 1 was defined as a "good response" to TACE. The predictive factors for the response to TACE were evaluated during CEUS based on the maximum tumor diameter, initial arterial enhancing time, arterial enhancing duration, intensity of arterial enhancement, presence of a hypoenhanced pattern, and the feeding artery to the tumor.

Results: The median tumor size was $3.1 \mathrm{~cm}$. The distribution of tumor response scores after TACE in all tumors was as follows: $1, n=11 ; 2, n=4 ; 3, n=2 ;$ and $4, n=1$. Fifteen tumors showed feeding arteries. The presence of a feeding artery and the tumor size $(\leq 5 \mathrm{~cm})$ were the predictive factors for a good response ( $P=0.043$ and $P=0.047$, respectively).

Conclusions: The presence of a feeding artery and a tumor size of less than $5 \mathrm{~cm}$ were the predictive factors for a good response of HCC to TACE on CEUS. (Clin Mol Hepatol 2015;21:158-164)

Keywords: Transarterial chemoembolization; Contrast-enhanced ultrasonography; Hepatocellular carcinoma; Tumor size; Feeding artery

\section{INTRODUCTION}

Hepatocellular carcinoma (HCC) is the fifth most frequently diagnosed cancer and the third cause of cancer-related mortality worldwide. ${ }^{1,2}$ Most patients with HCC are diagnosed late, and curative treatments cannot be applied. Additionally, high proportion of cases recur after curative therapy. ${ }^{3}$ Transarterial chemoembolization (TACE) is the most widely used primary treatment for unre-

\footnotetext{
Abbreviations:

CEUS, contrast-enhanced ultrasonography; CT, computed tomography; DSA, digital subtraction angiography; HCC, hepatocellular carcinoma; KLCSG-NCC, Korean Liver Cancer Study Group-National Cancer Center; MI, mechanical index; mUICC, Modified Union for International Cancer Control; MRI, magnetic resonance imaging; TACE, transarterial chemoembolization; US, ultrasonography; UCA, ultrasound contrast agent
}

\author{
Corresponding author: Soung Won Jeong \\ Institute for Digestive Research, and Digestive Disease Center, \\ Department of Internal Medicine, Soonchunhyang University Hospital, \\ 59 Daesagwan-ro, Yongsan-gu, Seoul 140-743, Korea \\ Tel: +82-2-709-3076, Fax: +82-2-709-9696 \\ E-mail: jeongsw@schmc.ac.kr
}


sectable HCC, and is recommended as first-line treatment for patients at intermediate stage of the disease by Barcelona Clinic Liver Cancer (BCLC) Staging System (B). ${ }^{4}$ HCC exhibits intense neoangiogenic activity during its progression. ${ }^{5}$ The rationale for TACE is that the intra-arterial infusion of a chemotherapeutic agent followed by embolization of the tumor-feeding artery will result in a strong cytotoxic and ischemic effect. ${ }^{6,7}$ Therefore, identification of the correct tumor-feeding artery is of definite importance on superselective (chemo) embolization. Successful subsegmental TACE in patients with HCC is related to a high degrees of tumor necrosis, low recurrence rates and prolonged survival. ${ }^{8,9}$ While, incorrect selection and subsequent TACE of a normal artery adjacent to the HCC will only result in damage of the normal liver parenchyma. So it is essential to evaluate tumor vascularization and distribution before TACE and during follow-up. Contrast-enhanced ultrasonography (CEUS) is now recognized as a useful imaging tool for the noninvasive diagnosis of small newly detected liver nodules during HCC surveillance and is also useful for guidance and assessment after loco-regional therapy of $\mathrm{HCC}^{10,11}$ Additionally low mechanical index (MI) real-time ultrasound in combination with ultrasound contrast agent (UCA) allows the real-time assessment of tumor vascularity and enhancement during the different vascular phases (arterial, portal venous, and delayed phases) with better temporal resolution than with contrast-enhanced computed tomography (CT) or magnetic resonance imaging (MRI). ${ }^{12}$ However, the predictive role of CEUS before TACE has not yet been elucidated. So in this study, we investigated for the first time the predictive factors for the response of TACE using CEUS.

\section{MATERIALS AND METHODS}

\section{Patients and HCCs}

Consecutive 17 patients (13 men, 4 women; mean age: $60 \pm 12.1$ years) with HCC who underwent CEUS before TACE and were evaluated for the response of TACE between April 2010 and March 2012 at Soonchunhyang University Hospital, Seoul, Korea were investigated retrospectively. A total of 18 target HCCs from 17 patients were assessed for the possible predictive factors on CEUS to the response of TACE. All target lesions were clearly characterized in abdominal US (ultrasonography) and successfully identified with CEUS. Patients were diagnosed with HCC by percutaneous liver biopsy or by the presentation of typical HCC radiological features of a hypervascular lesion arising in the setting of cirrhosis with arterial phase enhancement and portal venous phase washout and/or an elevated serum alpha-fetoprotein level using Korean Liver Cancer Study Group-National Cancer Center (KLCSG-NCC) practice guidelines in $2014 .{ }^{13}$ HCC was staged by the Modified Union for International Cancer Control (mUICC) stage. ${ }^{14}$ The decision to offer TACE to patients with HCC was made by a multidisciplinary medical team including hepatologists, surgeons, medical oncologists, therapeutic and interventional radiologists. Candidacy for TACE was established using KLCSG-NCC practice guidelines in $2014 .^{13}$

\section{CEUS}

All target HCCs were studied with CEUS before TACE. The mean

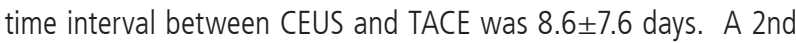
generation echo-enhancer SonoVue ${ }^{\circledR}$, a low MI technique and software dedicated to harmonic imaging in abdominal US were used. To investigate the predictive factors of CEUS for the response of TACE, maximal diameter of tumor, initial arterial enhancing time, arterial enhancing duration, the intensity of arterial enhancement (strong: whole tumor enhancement with high echo intensity, weak: whole or partial tumor enhancement with low echo intensity), presence of hypoenhanced pattern, and feeding artery to the tumor were evaluated during CEUS. All CEUS studies were performed by two experienced hepatologists.

\section{TACE}

A 5-French catheter was inserted into the common femoral artery and angiographic survey of the celiac and superior mesenteric arteries was performed. Digital subtraction angiography (DSA) was also performed after selective catheterization of the proper, right and/or left hepatic arteries. Vessels supplying one or two hepatic segments were selected with a coaxially placed microcatheter (Masters Parkway Soft, ASAHI, Aichi, Japan). Chemoembolization was performed with adriamycin mixed with lipiodol, which was infused into the probable tumor feeder through segmental/subsegmental hepatic artery. The administered doses of chemotherapeutic agents were adjusted in patients with tumor size (adriamycin $10 \mathrm{mg}$ per $1 \mathrm{~cm}$ diameter of tumor), liver or renal dysfunction, leukopenia, and thrombocytopenia. Administration of the emulsion was followed by embolization with a slurry of gelatin sponge (Cutanplast, Mascia Brunelli Spa, Milano, Italy; CaliGel, Alicon, Zhejiang, China) until stasis was achieved. TACE with drug-eluting beads were performed for two patients with HCC. 

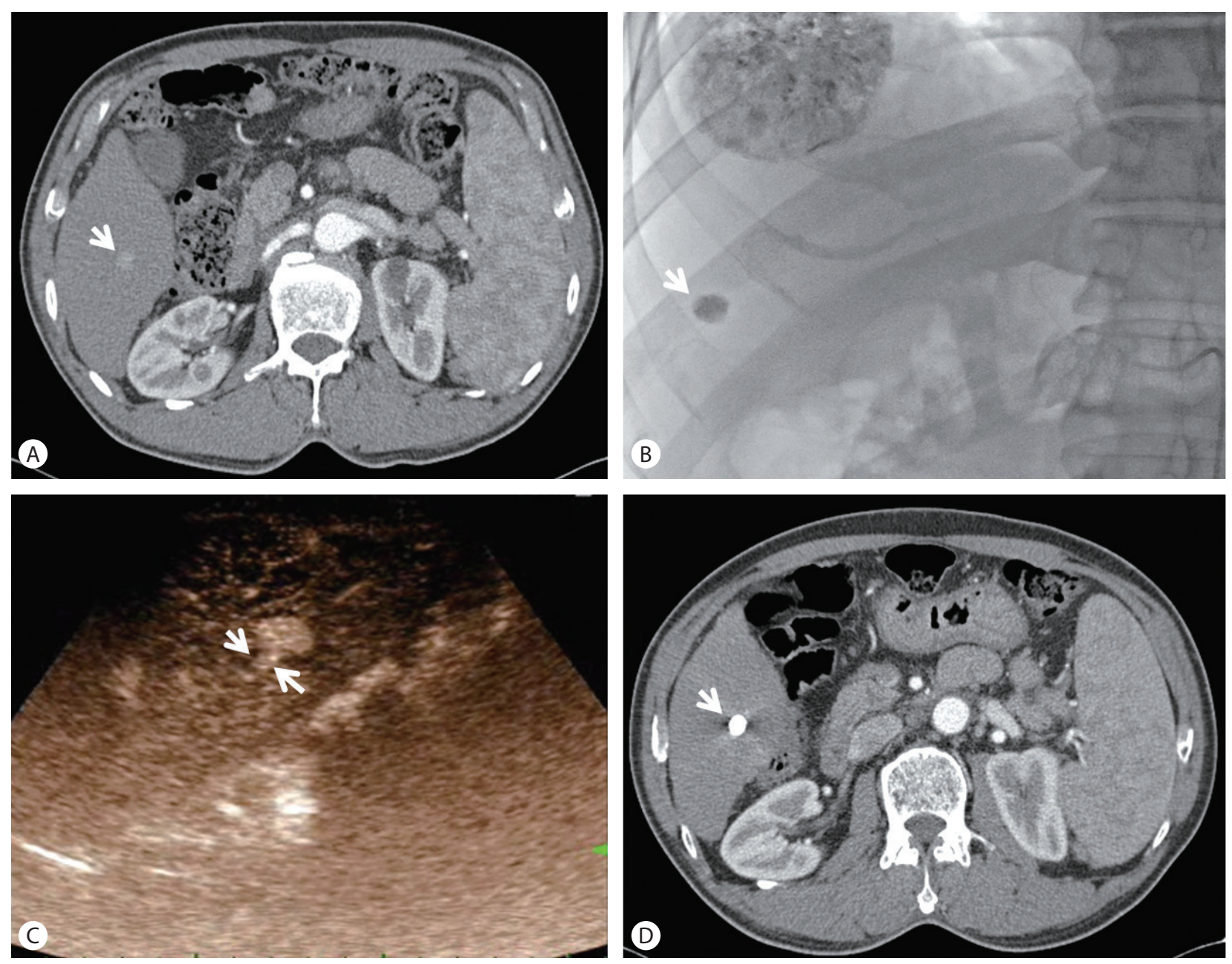

Figure 1. A tumor with a score of 1 after TACE. (A) An arrow indicates an enhancing HCC at arterial phase. (B) A tumor after TACE is indicated by an arrow. (C) Two arrows indicate the feeding artery to HCC in arterial phase of CEUS. (D) A compact lipiodol uptake after TACE is indicated by an arrow. TACE, transarterial chemoembolization; HCC, hepatocellular carcinoma; CEUS, contrast-enhanced ultrasonography.

The chemoembolic mixture consisted of a suspension of preloaded, drug-eluting microspheres (DC Beads Biocompatibles Ltd, Surrey, UK). DC Beads were preloaded with doxorubicin (Adriblastina, Pfizer Italia S.r.L., Nerviano, Milano, Italy) at a dose of $25-37.5 \mathrm{mg}$ drug/ml of hydrated beads. Each patient received 2-4 $\mathrm{mL}$ of DC Beads (diameters: 100-300 $\mu \mathrm{m}$ and 300-500 $\mu \mathrm{m}$ ).

\section{Post-TACE response assessment}

We assessed post-TACE response based on modified response evaluation criteria in solid tumors (mRECIST) ${ }^{15,16}$ tumor necrosis quantification on lipiodol contrast-enhanced CT which was underwent approximately 4 weeks after TACE. The mean time interval between TACE and lipiodol contrast-enhanced CT was $32 \pm 10.5$ days. After TACE, tumor response was classified with a score be- tween 1 and 4 according to the percentage of remaining enhancing tumor portion (score 1, remaining enhancing lesion $<25 \%$; score 2, 25\% senhancing lesion $<50 \%$; score 3, 50\% senhancing lesion $<75 \%$; score 4, enhancing lesion $\geq 75 \%$ ). Score 1 was defined as "good response" after TACE. We analyzed the correlation between post-TACE response score and tumor size, initial arterial enhancing time, arterial enhancing duration, intensity of arterial enhancement, presence of hypoenhanced pattern, and feeding artery to the tumor.

\section{Statistics}

Numerical data were expressed as mean value with \pm standard deviation or median value with range. The correlation between post-TACE response score and tumor size, initial arterial enhancing 
time, arterial enhancing duration, intensity of arterial enhancement, presence of hypoenhanced pattern, and feeding artery to the tumor were analyzed using Fisher's exact test. A $P$-value of $<0.05$ was considered significant. All statistical tests were 2-sided and performed with SPSS ver. 18.0 (SPSS Inc.,Chicago, IL, USA).

\section{RESULTS}

The demographics such as gender, age of the enrolled patient population and target HCC lesion characteristics such as causes, segmental distribution, mUICC stage, and Child-Pugh score are shown in Table 1. Features of target HCCs according to possible predictors on CEUS before TACE and post-TACE response scores were evaluated on the individual target HCC (Table 2). Median size of tumor was $3.1 \mathrm{~cm}$ (range 1.3-14 cm) and median initial arterial enhancing time was 21 seconds. Median arterial enhancing duration was 27.5 seconds and six tumors showed strong arterial enhancement. The presence of hypoenhancement pattern were 14 tumors. Fifteen tumors showed feeding arteries. The number of tumor response score after TACE in all tumors were 11 tumors with score 1, 4 with score 2, 2 with score 3, and 1 with score 4 . A tumor with score 1 is noted in Figure 1. In predicting good response after TACE, initial arterial enhancing time, arterial enhancing duration, intensity of arterial enhancement, and hypoenhanced pattern did not show any significance. Presence of a feeding artery and tumor size $(\leq 5 \mathrm{~cm})$ were predictive factors for good response, respectively $(P=0.043, P=0.047)$ (Table 3 ).

\section{DISCUSSION}

CEUS is a flexible and versatile tool to many hepatologists, playing an important role in therapeutic planning, interventional guidance and post-treatment evaluation. We investigated the predictive factors for the response of TACE using CEUS for the first time.

And we used the low $\mathrm{Ml}$ real-time ultrasound in combination with second generation UCA SonoVue ${ }^{\circledR}$ and software dedicated to harmonic imaging for the evaluation of predictive factors with the following reasons. First, physically the generation of harmonics in CEUS is related to nonlinearly (squared relation between second harmonic and applied acoustic pressure) to fundamental

Table 1. Patient demographics and characteristics of target HCCs

\begin{tabular}{|c|c|c|c|c|c|}
\hline No & $\begin{array}{l}\text { Gender } \\
\text { /Age }\end{array}$ & $\begin{array}{l}\text { Segmental } \\
\text { distribution }\end{array}$ & $\begin{array}{c}\text { Cause of } \\
\text { HCC }\end{array}$ & Modified UICC stage & Child-Pugh score \\
\hline 1 & $M / 44$ & S6 & HBV & III & 6 \\
\hline 2 & $M / 62$ & 56 & $\mathrm{HCV}$ & 1 & 6 \\
\hline 3 & $M / 57$ & $\mathrm{~S} 5,6$ & HBV & IV B & 6 \\
\hline 4 & $\mathrm{M} / 72$ & S5 & $\mathrm{HCV}$ & $\|$ & 5 \\
\hline 5 & $F / 49$ & S5 & HBV & IV A & 6 \\
\hline 6 & $\mathrm{~F} / 60$ & S6 & HBV & । & 5 \\
\hline 7 & M/39 & S5 & Alcohol & III & 5 \\
\hline 8 & $\mathrm{M} / 40$ & S7 & HBV & III & 6 \\
\hline 9 & $\mathrm{M} / 81$ & $\$ 4,6,8$ & HBV & III & 5 \\
\hline 10 & $M / 59$ & S6 & HBV & IV A & 7 \\
\hline 11 & $F / 73$ & S6 & HBV & IV A & 6 \\
\hline 12 & $M / 56$ & $S 5,6,7,8$ & HBV & IV B & 5 \\
\hline 13 & $M / 63$ & 56 & HBV & $\|$ & 8 \\
\hline 14 & $M / 50$ & S4 & Cryptogenic & $\|$ & 5 \\
\hline 15 & $\mathrm{~F} / 71$ & S6 & $\mathrm{HCV}$ & $\|$ & 6 \\
\hline 16 & M/62 & $\mathrm{S} 2$ & $\mathrm{HBV}$ & III & 5 \\
\hline 17 & M/71 & $\mathrm{S} 6$ & HBV & III & 5 \\
\hline 18 & $M / 71$ & S6 & HBV & III & 5 \\
\hline
\end{tabular}

HCC, hepatocellular carcinoma; M, male; F, female; S, segment; TACE, transarterial chemoembolization; HBV, hepatitis B virus; HCV, hepatitis C virus; UICC, Union for International Cancer Control. 
Table 2. Features of target HCCs according to possible predictors

\begin{tabular}{|c|c|c|c|c|c|c|c|}
\hline No & $\begin{array}{l}\text { The intensity of } \\
\text { arterial } \\
\text { enhancement }\end{array}$ & $\begin{array}{l}\text { Feeding artery } \\
\text { (presence } \\
\text { /number) }\end{array}$ & $\begin{array}{l}\text { Maximal } \\
\text { diameter } \\
(\mathrm{cm})\end{array}$ & $\begin{array}{c}\text { Initial arterial } \\
\text { enhancing time } \\
\text { (second) }\end{array}$ & $\begin{array}{c}\text { Presence of } \\
\text { hypoenhanced } \\
\text { pattern }\end{array}$ & $\begin{array}{l}\text { Arterial enhancing } \\
\text { duration } \\
\text { (second) }\end{array}$ & $\begin{array}{l}\text { Response } \\
\text { score }^{*}\end{array}$ \\
\hline 1 & Weak & No (0) & 2.0 & 18 & No & 42 & 2 \\
\hline 2 & Strong & Yes (1) & 1.5 & 30 & No & 40 & 1 \\
\hline 3 & Weak & No (0) & 3.3 & 28 & No & 52 & 4 \\
\hline 4 & Weak & Yes (2) & 3.1 & 22 & Yes & 23 & 1 \\
\hline 5 & Strong & Yes (1) & 1.3 & 9 & Yes & 21 & 1 \\
\hline 6 & Weak & Yes (1) & 2.0 & 20 & Yes & 20 & 1 \\
\hline 7 & Weak & Yes (1) & 2.7 & 13 & Yes & 20 & 1 \\
\hline 8 & Weak & Yes (1) & 3.0 & 22 & Yes & 28 & 1 \\
\hline 9 & Strong & Yes (1) & 10 & 15 & Yes & 45 & 2 \\
\hline 10 & Strong & $\mathrm{No}(0)$ & 6.6 & 15 & Yes & 27 & 3 \\
\hline 11 & Strong & Yes (1) & 7.0 & 23 & Yes & 29 & 2 \\
\hline 12 & Weak & Yes (2) & 14 & 13 & Yes & 25 & 3 \\
\hline 13 & Strong & Yes (1) & 5.6 & 45 & Yes & 27 & 1 \\
\hline 14 & Weak & Yes (1) & 5.0 & 25 & Yes & 35 & 1 \\
\hline 15 & Weak & Yes (1) & 2.0 & 25 & No & 35 & 1 \\
\hline 16 & Weak & Yes (4) & 10 & 25 & Yes & 45 & 2 \\
\hline 17 & Weak & Yes (3) & 2.8 & 20 & Yes & 15 & 1 \\
\hline 18 & Weak & Yes (3) & 3.4 & 17 & Yes & 7 & 1 \\
\hline Total & $\begin{array}{l}\text { Strong (6), } \\
\text { weak (12) }\end{array}$ & $\begin{array}{c}\text { Yes (15) } \\
\text { No (3) }\end{array}$ & $\begin{array}{c}3.1^{\dagger} \\
(1.3-10)^{\ddagger}\end{array}$ & $\begin{array}{c}21^{\dagger} \\
(9-45)^{\ddagger}\end{array}$ & Yes (14) No (4) & $\begin{array}{c}27.5^{\dagger} \\
(7-52)^{\ddagger}\end{array}$ & $\begin{array}{c}1 / 2 / 3 / 4 \\
(11 / 4 / 2 / 1)\end{array}$ \\
\hline
\end{tabular}

HCC, hepatocellular carcinoma.

"Score 1, remaining enhancing lesion <25\%; score 2, 25\% senhancing lesion<50\%; score 3, 50\% senhancing lesion< $75 \%$; score 4, enhancing lesion $\geq 75 \%$ ).

${ }^{\dagger}$ Median.

${ }^{\ddagger}$ Range.

Table 3. Predictive factors for a good response of HCC to TACE on CEUS

\begin{tabular}{lc}
\hline Characteristics & $\boldsymbol{P}$-value \\
\hline Tumor size $(\leq 5 \mathrm{~cm})$ & 0.047 \\
Initial arterial enhancing time $(\leq 21 \mathrm{sec})$ & 1.000 \\
\hline Arterial enhancing duration $(\leq 28 \mathrm{sec})$ & 0.335 \\
Intensity of arterial enhancement & 0.627 \\
Presence of hypoenhanced pattern & 1.000 \\
\hline Feeding artery to the tumor & 0.043 \\
\hline
\end{tabular}

TACE, transarterial chemoembolization; CEUS, contrast-enhanced ultrasonography.

frequency energy. ${ }^{17}$ Imaging in the harmonic range will eliminate much of the near-filed artifacts such as reverberation and scattering. So it allows intravascular image of high-resolution during entire vascularphase. ${ }^{18}$ Second, current UCAs are typically microbubbles encapsulated by a stabilizing shell such as albumin, polymer, or phospholipid. Microbubbles are smaller than red blood cells (up to $7 \mu \mathrm{m}$ in diameter), so they can easily pass through the capillary beds. On the contrary, they are too big to escape through the vascular endothelial pore unlike contrast agent of CT or MRI, thereby acting as excellent blood pool tracers. ${ }^{19}$ Third, at low acoustic peak pressure levels (low MI), microbubbles don't burst but oscillate (due to blood circulation) during enough vascularphase unlike first-generation UCA using high $\mathrm{MI}^{20}$ Therefore, the above imaging tool facilitates real-time vascularphase (i.e., in the arterial, portal venous, and delayed phases) imaging of high-resolution for several minutes. ${ }^{21,22}$

CEUS has another several advantages to assess the predictive factors for the response of TACE comparing with contrast-enhanced CT and MRI. CEUS is real-time imaging, whereas both contrast-enhanced CT and MRI are static imaging during each of the phases of enhancement. Some HCCs show transient hypervascularity in the arterial phase of real time CEUS, however they do 
not show enhancement in contrast-enhanced CT and MRI. ${ }^{23,24}$ So we can infer that CEUS is more effective for showing feeding artery than contrast-enhanced CT and MRI. Additionally, CEUS can be used without risk of nephrotoxicity or requirement for ionizing radiation.

However, CEUS has disadvantages against contrast-enhanced CT and MRI. It cannot exhibit comprehensive assessment of the whole liver parenchyma during the short duration of the arterial phase. ${ }^{25}$ Another limitation is that like basic ultrasound techniques, CEUS can't get clear imaging in patients with a poor acoustic window including deep seated lesion. ${ }^{26}$ In addition, if multiple lesions or very large tumor are present, the lesions or the whole tumor can't be captured on single plane. Therefore, contrast-enhanced CT or MRI is effective for comprehensive assessment of the whole liver before TACE, but CEUS can be effective for prediction of the response of the target HCC.

According to the result of this study, initial arterial enhancing time, arterial enhancing duration, intensity of arterial enhancement, and hypoenhanced pattern did not show any significance, whereas presence of a feeding artery and tumor size $(\leq 5 \mathrm{~cm})$ were predictive factors for good response, respectively.

Presence of a feeding artery was the significant predictor for good response of TACE and this result confirms that above all, identification of the correct tumor-feeding artery is of definite importance for the good response of TACE. ${ }^{8,9}$ Kwan et al ${ }^{27}$ verified that the presence of a tumor-feeding artery greater than $0.9 \mathrm{~mm}$ in diameter on the pre-TACE visceral angiogram is a significant favorable predictor of $>90 \%$ TACE-induced tumor necrosis. The correlation between definite presence of feeding artery on CEUS and definite presence of tumor-feeding artery on pre-TACE visceral angiogram or DSA needs to be further investigated. And if the concordance can be verified, further research will be required for confirm the efficacy of contrast-enhanced ultrasound with intraarterial administration of UCA (i.a CEUS) guidance, which distinguish and help identify correct tumor-feeding artery in HCC with a complex vascular supply, or in small and hypovascular HCC, with little or no tumor blush on DSA during TACE. ${ }^{28}$ In this study, tumor size $(\leq 5 \mathrm{~cm})$ was also significant factor for favorable response. In terms of tumor size, the large size tumor generally has multiple feeding arteries and it seems that it is more difficult to embolize all feeding arteries and make large tumor be necrotized.

But, this study has several limitations. First, large HCC with multiple feeding arteries might be not captured on single plane at the same time during arterial enhancement. Second, there was a little bit of range for time interval between CEUS and TACE, and also the time interval between TACE and lipiodol contrast-enhanced CT. Third, the retrospective nature and small number of target HCCs may be potential limitations of this study. So to validate the result of this study, there are need for large sized population and prospective design.

In conclusion, this study showed real-time imaging of CEUS can effectively predict the response of TACE for target HCC, and the presence of a feeding artery and tumor size less than $5 \mathrm{~cm}$ were the predictive factors for the good response of TACE on CEUS.

\section{Conflicts of Interest}

The authors have no conflicts to disclose.

\section{REFERENCES}

1. Forner A, Llovet JM, Bruix J. Hepatocellular carcinoma. Lancet 2012:379:1245-1255

2. Tsochatzis EA, Bosch J, Burroughs AK. Liver cirrhosis. Lancet 2014;383:1749-1761.

3. Lencioni R. Chemoembolization for hepatocellular carcinoma. Semin Oncol 2012;39:503-509.

4. Tsochatzis EA, Germani G, Burroughs AK. Transarterial chemoembolization, transarterial chemotherapy, and intra-arterial chemotherapy for hepatocellular carcinoma treatment. Semin Oncol 2010;37:89-93.

5. Fatourou EM, Koskinas JS. Adaptive immunity in hepatocellular carcinoma: prognostic and therapeutic implications. Expert Rev Anticancer Ther 2009;9:1499-1510.

6. Tsochatzis EA, Fatourou E, O'Beirne J, Meyer T, Burroughs AK. Transarterial chemoemboliza-tion and bland embolization for hepatocellular carcinoma. World J Gastroenterol 2014;20:3069-3077.

7. Tsochatzis EA, Fatourou EM, Triantos CK, Burroughs AK. Transarterial therapies for hepato-cellular carcinoma. Recent Results Cancer Res 2013;190:195-206.

8. Matsui O, Kadoya M, Yoshikawa J, Gabata T, Arai K, Demachi H, et al. Small hepatocellular carcinoma: treatment with subsegmental transcatheter arterial embolization. Radiology 1993;188:79-83.

9. Iwamoto S, Sanefuji H, Okuda K. Angiographic subsegmentectomy for the treatment of patients with small hepatocellular carcinoma. Cancer 2003;97:1051-1056

10. Schneider M. Characteristics of SonoVuetrade mark. Echocardiography 1999;16:743-746.

11. Nicolau C, Vilana R, Catalá V, Bianchi L, Gilabert R, García A, et al. Importance of evaluating all vascular phases on contrast-enhanced sonography in the differentiation of benign from malignant focal liver lesions. AJR Am J Roentgenol 2006;186:158-167.

12. Trillaud H, Bruel JM, Valette PJ, Vilgrain V, Schmutz G, Oyen R, et al. 
Characterization of focal liver lesions with SonoVue-enhanced sonography: international multicenter-study in comparison to CT and MRI. World J Gastroenterol 2009;15:3748-3756.

13. Lee JM, Park JW, Choi BI. 2014 KLCSG-NCC Korea Practice Guidelines for the management of hepatocellular carcinoma: HCC diagnostic algorithm. Dig Dis 2014;32:764-777.

14. Ueno S, Tanabe G, Nuruki K, Hamanoue M, Komorizono Y, Oketani $M$, et al. Prognostic per-formance of the new classification of primary liver cancer of Japan (4th edition) for patients with hepatocellular carcinoma: a validation analysis. Hepatol Res 2002;24:395-403.

15. Lencioni R. Management of hepatocellular carcinoma with transarterial chemoembolization in the era of systemic targeted therapy. Crit Rev Oncol Hematol 2012;83:216-224.

16. Lencioni $R$, Crocetti L. Local-regional treatment of hepatocellular carcinoma. Radiology 2012;262:43-58.

17. Adler $L$, Hiedemann E. Determination of the Nonlinearity Parameter B/A for Water and m-Xylene. J Acoust Soc Am 1962;34:410-412.

18. Thomas JD, Rubin DN. Tissue harmonic imaging: why does it work? J Am Soc Echocardiogr 1998;11:803-808.

19. Piscaglia F, Lencioni R, Sagrini E, Pina CD, Cioni D, Vidili G, et al. Characterization of focal liver lesions with contrast-enhanced ultrasound. Ultrasound Med Biol 2010;36:531-550.

20. Harvey CJ, Blomley MJ, Eckersley RJ, Heckemann RA, Butler-Barnes J, Cosgrove DO. Pulse-inversion mode imaging of liver specific microbubbles: improved detection of subcentimetre metastases. Lancet 2000;355:807-808.

21. Bolondi L, Correas JM, Lencioni R, Weskott HP, Piscaglia F. New per- spectives for the use of contrast-enhanced liver ultrasound in clinical practice. Dig Liver Dis 2007;39:187-195.

22. Lencioni $R$, Cioni $D$, Bartolozzi C. Tissue harmonic and contrastspecific imaging: back to gray scale in ultrasound. Eur Radiol 2002;12:151-165.

23. Jang JY, Kim MY, Jeong SW, Kim TY, Kim SU, Lee SH, et al. Current consensus and guidelines of contrast enhanced ultrasound for the characterization of focal liver lesions. Clin Mol Hepatol 2013;19:1-16.

24. Wilson SR, Kim TK, Jang HJ, Burns PN. Enhancement patterns of focal liver masses: discord-ance between contrast-enhanced sonography and contrast-enhanced CT and MRI. AJR Am J Roentgenol 2007;189:W7-W12.

25. Lencioni R, Piscaglia F, Bolondi L. Contrast-enhanced ultrasound in the diagnosis of hepatocel-Iular carcinoma. J Hepatol 2008;48:848-857.

26. Strobel D, Seitz K, Blank W, Schuler A, Dietrich C, von Herbay A, et al. Contrast-enhanced ultrasound for the characterization of focal liver lesions--diagnostic accuracy in clinical practice (DEGUM multicenter trial). Ultraschall Med 2008;29:499-505.

27. Kwan SW, Fidelman N, Ma E, Kerlan RK Jr, Yao FY. Imaging predictors of the response to transarterial chemoembolization in patients with hepatocellular carcinoma: a radiological-pathological correlation. Liver Transpl 2012;18:727-736.

28. Moschouris H, Malagari K, Kalokairinou M, Stamatiou K, Marinis A, Papadaki MG. Contrast-enhanced ultrasonography with intraarterial administration of SonoVue for guidance of transarterial chemoembolization: an initial experience. Med Ultrason 2011;13:296301. 